\title{
Polarity Control and Nanoscale Optical Characterization of AlGaN-based Multiple Quantum Wells for Ultraviolet C emitters
}

Houqiang Xu ${ }^{1,2}$, Jie'an Jiang ${ }^{1,2}$, Yijun Dai ${ }^{1,2}$, Mei Cui ${ }^{1,2}$, Kuang-hui Li' ${ }^{3}$, Xiaotian Ge ${ }^{4}$, Jason Hoo ${ }^{5}$, Long Yan ${ }^{5}$, Shiping Guo ${ }^{5}$, Jiqiang Ning 4 , Haiding Sun ${ }^{6}$, Biplab Sarkar , Wei Guo ${ }^{1,2 *}$, and Jichun $Y e^{1,2 *}$

${ }^{1}$ Ningbo Institute of Materials Technology and Engineering, Chinese Academy of Sciences, Ningbo, 315201, Zhejiang, China

${ }^{2}$ University of Chinese Academy of Sciences, Beijing, 100049, China

${ }^{3}$ King Abdullah University of Science and Technology (KAUST), Thuwal 23955-6900, Saudi Arabia

${ }^{4}$ Vacuum Interconnected, Nanotech Workstation, Suzhou Institute of Nano-Tech and Nano-Bionics (SINANO), Suzhou, 215123, Jiangsu, China

${ }^{5}$ Advanced Micro-Fabrication Equipment Inc. Shanghai, 201201, China

${ }^{6}$ School of Microelectronics, University of Science and Technology of China, Hefei, 230026, Anhui, China

${ }^{7}$ Department of Electronics and Communication Engineering, IIT Roorkee, Roorkee, Uttarakhand 247667, India

Email: guowei@nimte.ac.cn,jichun.ye@nimte.ac.cn 


\begin{abstract}
Optical properties of AlGaN UVC multiple-quantum-wells (MQWs) with nanoscale inverted polarity domains are strongly related to the polar surfaces and nanoscale structures. In this work, the impact of pre-growth nitridation of the sapphire substrate on the polarity control of UVC MQW is highlighted and the optical properties of III- and N-polar domains were distinguished. Nanoscale cathodoluminescence peak separation of more than $30 \mathrm{~nm}$ is observed in lateral-polarity-structure (LPS) UVC MQWs, which is ascribed to the potential minima induced by local variation of QW thickness and Ga enrichment inside N-polar domains. After inserting an AlGaN/AlN superlattice and enhancing V/III ratio during growth, the surface morphology of N-polar domain is greatly improved, leading to a single peak emission at wavelength of $275 \mathrm{~nm}$ in both III- and N-polar domains, and 10-fold stronger peak intensity at the inversion domain boundary. Such understandings on the polar surface optimization and underlying reasons of peak separation enable rational design for efficient UVC emitters with improved performance.
\end{abstract}

Keywords: AlGaN, UVC-LED, polarity control, luminescence property, carrier localization

\title{
1. Introduction
}

With increasing demands in applications of ultraviolet (UV) light including UV curing, disinfection, water purification and photocuring, researchers have paid great attention in the development of solid-state UV emitters like UV light-emitting-diodes 
(UV-LEDs) to replace the conventional bulky, low-efficiency and toxic mercury lamps. ${ }^{1,2}$ However, the commercial applications of UV LEDs, especially those with emission wavelength shorter than $280 \mathrm{~nm}$ (i.e. in UVC region) are still hindered by their relatively low external quantum efficiency (EQE) mainly owing to poor crystalline quality of $\mathrm{AlGaN}$ thin film ${ }^{3}$ and total internal reflection at the interface between nitrides and air. ${ }^{4}$ As a consequence, the highest EQE value of UVC LED in records is $20 \%$ so far, which is far less than that of the InGaN/GaN-based blue LED counterpart. ${ }^{5,6}$ In order to obtain high-efficiency UVC LED, many attempts have been applied, such as utilization of nanoscale patterned sapphire substrate during epitaxial growth, ${ }^{7}$ thin film epitaxy on high misoriented sapphire substrate, ${ }^{8}$ fabrication of photonic crystal and implementation of surface plasmon polaritons on the devices ${ }^{9}$ to efficiently extract photons, but limited success was achieved, due to poor understanding on the relationship between optical properties and nanoscale structure and composition analysis in the active region of the UVC-LEDs.

Recently, a unique structure of GaN/AlGaN UVA multiple-quantum-wells (MQWs) emitting at $360 \mathrm{~nm}$ consisting of both nanoscale III and N-polar domains was fabricated by our group. ${ }^{10}$ This so-called "lateral polarity structure (LPS)" enables rich functionalities in the application of optoelectronic devices by inheriting the advantages of both III- and N-polar surfaces of III-nitride crystals. Unlike conventional c-plane MQW in which light emissions suffer from strong quantum-confined-stark-effect, ${ }^{11}$ carriers transport and recombination in a three-dimensional configuration inside this LPS-based MQW due to the opposite 
directions of band bending in III- and N-polar domains. ${ }^{12}$ Thanks to this unique energy band structure, 6-fold enhancement in light emission was demonstrated compared to conventional single-polarity MQWs. However, with increasing Al composition in the MQWs, the development of UVC-LEDs faces two obstructing limitations. Firstly, as the growth of high Al-content AlGaN follows 3D columnar mode, deterioration of surface morphology and crystalline quality is expected, preventing the formation of abrupt domain boundaries and efficient carrier recombination. ${ }^{13}$ Secondly, as $\mathrm{Al}$ and Ga have drastic different surface diffusion lengths during epitaxial growth, lateral atom migration might occur across domain boundary, leading to emission peak separation and composition deviation from the designed structure. A deeper understanding of these challenges is strongly desired and would promote the wide application of LPS in III-nitride UVC photonic devices.

In this work, growth optimization and nanoscale optical characterizations were performed in order to break the conventional limit of peak separation and poor recombination efficiency of LPS-based $\mathrm{Al}_{\mathrm{x}} \mathrm{Ga}_{1-\mathrm{x}} \mathrm{N} / \mathrm{Al}_{\mathrm{y}} \mathrm{Ga}_{1-\mathrm{y}} \mathrm{N}$ UVC MQWs. The influences of pre-growth nitridation and epitaxy parameters on the modulation of thin film polarities were thoroughly discussed. The underlying reasons for the existence of double peaks (280 nm and $314 \mathrm{~nm}$ ) shown in UVC MQWs were analyzed in detail. Furthermore, by growth modification, surface morphologies of the LPS-based UVC MQWs were improved, bimodal phase separation between III- and N-polar domains were therefore eliminated and single-peak luminescence with 10-fold enhancement in the domain boundary was achieved. 


\section{Experimental Section:}

$\mathrm{Al}_{0.55} \mathrm{Ga}_{0.45} \mathrm{~N} / \mathrm{Al}_{0.4} \mathrm{Ga}_{0.6} \mathrm{~N}$ MQWs were grown on 2 inch $c$-plane sapphire substrates using an AMEC Prismo $\mathrm{HiT}_{3}{ }^{\mathrm{TM}}$ MOCVD reactor in $\mathrm{H}_{2}$ ambient. Trimethylaluminum, trimethylgallium and ammonia $\left(\mathrm{NH}_{3}\right)$ were used as precursors of $\mathrm{Al}, \mathrm{Ga}$ and $\mathrm{N}$, respectively. Hydrogen $\left(\mathrm{H}_{2}\right)$ and nitrogen $\left(\mathrm{N}_{2}\right)$ were used as the carrier gases. Initially, $20 \mathrm{~nm}$ thick LT-AlN buffer was deposited on the sapphire substrate at $850{ }^{\circ} \mathrm{C}$. LT-AIN was then patterned through photolithography followed by wet etching. Exposed LT-AlN buffer was completely removed and buffer underneath photoresist remained intact. After a thorough cleaning step, the patterned AlN buffer was re-loaded into the MOCVD chamber. Thermal annealing under $\mathrm{NH}_{3}$ atmosphere was performed under various temperatures. $2.8 \mu \mathrm{m}$ HT-AlN template was subsequently grown under $1250{ }^{\circ} \mathrm{C}$, followed by $1.5 \mu \mathrm{m}$ thick $\mathrm{Al}_{0.6} \mathrm{Ga}_{0.4} \mathrm{~N}$ transition layer, and eight pairs of $\mathrm{Al}_{0.55} \mathrm{Ga}_{0.45} \mathrm{~N} / \mathrm{Al}_{0.4} \mathrm{Ga}_{0.6} \mathrm{~N}$ MQWs $(8 \mathrm{~nm} / 3 \mathrm{~nm})$. Finally, $20 \mathrm{~nm} \mathrm{Al}_{0.6} \mathrm{Ga}_{0.4} \mathrm{~N}$ cap layer was deposited on the surface. Surface morphologies of these samples were characterized by Hitachi S-4800 field-emission SEM and Veeco Dimension $3100 \mathrm{~V}$ AFM. Crystalline qualities were analyzed using a point-focused HRXRD (Bruker D8 DISCOVER) equipped with a four-bounce symmetric Ge (220) monochromator. RT-PL studies were performed using a Coherent Ar-F (193 nm) excimer laser at

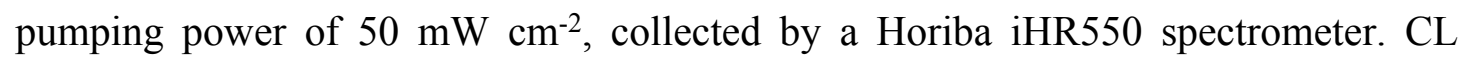
investigations were undertaken using a Horiba MP 325 CL characterization system with voltage of $5 \mathrm{kV}$ and current level of $188 \mu \mathrm{A}$. TEM samples were prepared using an FEI Helios dual-beam focused ion beam scanning electron microscope system with 
a Ga ion source. Cross-sectional morphology of both III- and N-polar domains, MQW layer thickness, as well as dislocation distribution were characterized by a FEI probe-corrected HAADF-STEM operated at an acceleration voltage of $300 \mathrm{kV}$.

\section{Results and discussion:}

AlGaN UVC-MQWs were grown on 2 inch $c$-plane sapphire substrates and the fabrication process scheme is shown in Figure 1. The fabrication of LPS depends on patterning of underlying LT-AIN buffer deposited on sapphire. Generally, III-nitride thin film grown on AlN buffer leads to III-polarity while film grown on bare sapphire with proper nitridation results in N-polarity. ${ }^{14}$ However, the control of thin film quality and interface abruptness of LPS especially high-Al-content AlGaN-based LPS is not trivial. Prior to high-temperature epitaxial growth, the patterned AlN buffer went through $\mathrm{H}_{2}$ annealing followed by nitridation process under $\mathrm{NH}_{3}$ atmosphere. Different nitridation temperatures including $950{ }^{\circ} \mathrm{C}, 1050{ }^{\circ} \mathrm{C}$ and $1150{ }^{\circ} \mathrm{C}$ were adopted in this work, and the corresponding samples are labeled as sample A, B and C respectively.

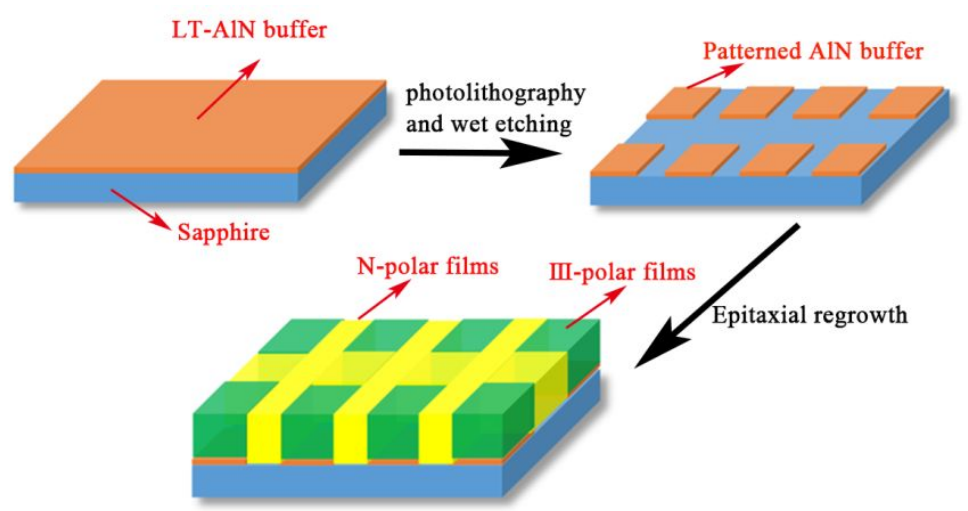

Figure 1. The fabrication process scheme of LPS-based MQWs. The process contains three main steps. Step 1: MOCVD deposition of LT-AlN buffer on c-plane sapphire. 
Step 2: Fabricating patterned buffer through photolithography and wet etching. Step 3: The patterned substrate is reloaded into the MOCVD chamber for epitaxial regrowth.

$\mathrm{KOH}$ etching was performed at $70^{\circ} \mathrm{C}$ for $10 \mathrm{~min}$ to verify the polarities of the patterned domains of AlGaN-MQWs with different nitridation conditions. ${ }^{15}$ It is known that the chemical stability of III-nitride thin film is related to its surface energy, which is a function of the dangling bond density. Therefore, N-polar surfaces are more easily etched than III-polar surfaces under alkaline solution due to much higher density of dangling bonds. ${ }^{16}$ Top-view SEM images of the as-grown sample A, $\mathrm{B}$ and $\mathrm{C}$ are shown in Figure 2(a)-(c), and corresponding images after chemical etching are shown in Figure 2(d)-(f). The domain on the left-hand side of each sample is grown on AlN buffer, whereas domain on the right-hand side is grown on bare sapphire. It's noticed that the morphologies on the left hand side of each image remain smooth regardless of nitridation temperature, confirming the nature of III-polarity. The morphologies on the right hand side of each image are distinctively different. Sample A shows wrinkle-like morphology, where majority of the surface is dominated with $+c$ crystallographic planes. As the nitridation temperature increases, the surfaces exhibit flake-like semipolar planes as shown in Figure 2(b) and (c). After $\mathrm{KOH}$ etching, domains on the left hand side of each image are unaffected, but domains on the right exhibit different etching behaviors. Sample A is dominant with hexagonal or round pits-like features with pinholes located at the bottoms, which is an 
indication of mix-polar surface and insufficient nitridation. While for sample B, after 10 min etching process, the domain boundary appears very sharp and the N-polar domain is flat without any residual hillocks, indicating that thin films on the right hand side are completely removed, demonstrating the formation of pure N-polar surfaces. For Sample C, surface morphology after etching is similar to that of Sample B, except for rougher exposed sapphire substrate with small pits as indicated by the arrows, and occurrence of mix-polar transition region at the domain boundary as indicated by the red dashed box. Note that strong chemical reaction between sapphire and $\mathrm{NH}_{3}$ can cause local decomposition of sapphire. ${ }^{17}$ Therefore, the high density of pits on sapphire substrate in Figure 2(f) is a strong indication of over-nitridation, which could lead to poor crystalline quality and rough MQW morphologies that contain both polar and semi-polar crystal planes. The mix-polar domains at the domain boundary can be explained by the fact that, after intense nitridation process, AlN buffer near the domain boundaries was partially damaged, leading to the formation of irregular mixed polar domains of several micrometers wide. Even though the detailed mechanism is still under debate, researchers claimed that the transformation of $\mathrm{Al}_{2} \mathrm{O}_{3}$ into $\mathrm{AlN}$ on the very top surface of sapphire is critical during polarity control. ${ }^{18}$ A higher nitridation temperature can promote the formation of N-polarity, however, at the expense of sapphire decomposition as mentioned in the manuscript. Thus the nitridation condition (e.g., temperature and time) is vital for polarity control. It can be safely concluded that polarity control was successfully 
achieved in sample $\mathrm{B}$, and the optimal nitridation condition is obtained at $1050{ }^{\circ} \mathrm{C}$, which is critical for polarity control in LPS samples.
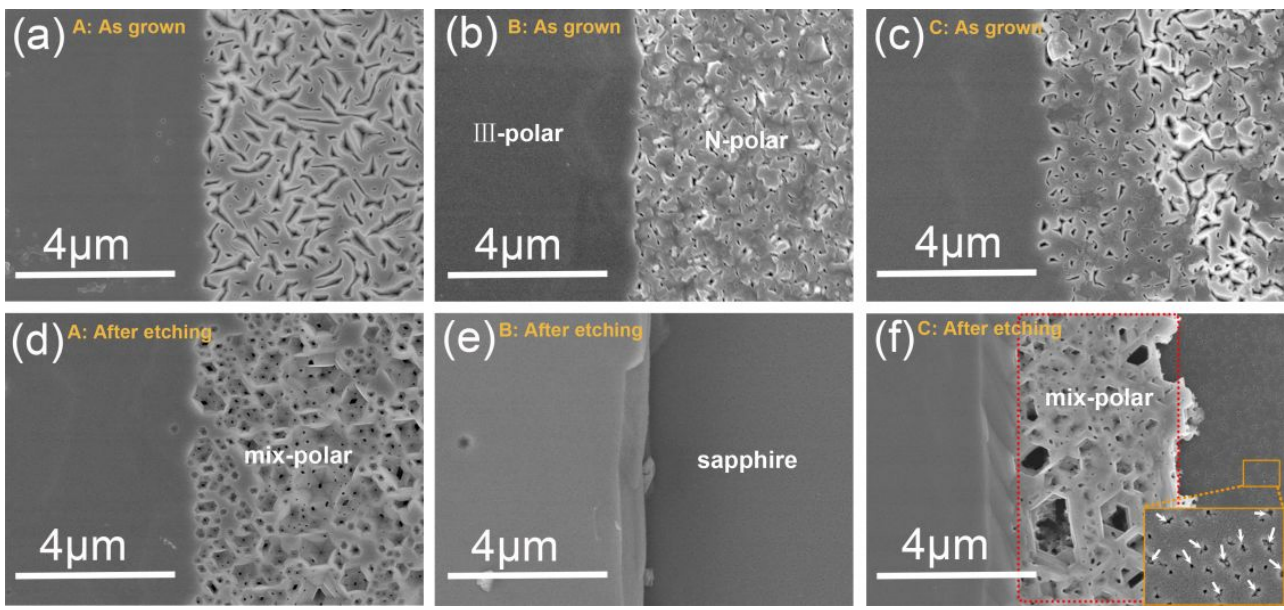

Figure 2. Top-view SEM images of sample A, B and C before (a-c) and after (d-f) $\mathrm{KOH}$ etching for $10 \mathrm{~min}$. The left side of each image is III-polar domain, and right side of each image is designated for N-polar domain.

High-resolution X-ray diffraction (HRXRD) rocking curves (RCs) of the (002) and (102) reflections of sample B in both III- and N-polar domains were performed to characterize the dislocation density. The reflections are taken from AlGaN template due to its higher intensity compared to MQW region. As shown in Figure S1, FWHM of (002) X-ray RC of N-polar domain is almost two-times higher than that of III-polar domain, indicating higher threading dislocation density in the former region. ${ }^{19}$ The edge and screw type dislocation densities are calculated to be $4.55 \times 10^{8} \mathrm{~cm}^{-2}$, $3.18 \times 10^{10} \mathrm{~cm}^{-2}$ for III-polar domain. The corresponding values are $1.91 \times 10^{9} \mathrm{~cm}^{-2}$ and $3.47 \times 10^{10} \mathrm{~cm}^{-2}$ for N-polar domain.

Different surface morphologies and crystalline quality between III- and N-polar MQWs are directly correlated with their optical properties. Herein, room temperature 
(RT) photoluminescence (PL) of the optimized LPS MQW (sample B) was collected and illustrated in Figure 3. The schematic structure of the MQWs with laterally-distributed III- and N-polar domains is shown in the inset image. The periodicity of the alternating domain is $100 \mu \mathrm{m}$, and the illumination area of the laser spot is approximately $1 \times 1 \mathrm{~mm}^{2}$, therefore enable simultaneous illumination of both the III- and N-polar domains. The PL spectrum indicates two distinct peaks at $280 \mathrm{~nm}$ and $314 \mathrm{~nm}$, with the latter one being much stronger, and a large peak separation of $34 \mathrm{~nm}$ is obtained. Note that the wavelength at $280 \mathrm{~nm}$ is the target emission of the MQWs. Therefore, the much longer $314 \mathrm{~nm}$ emission peak may originate from carrier localization caused by rough surface morphology.

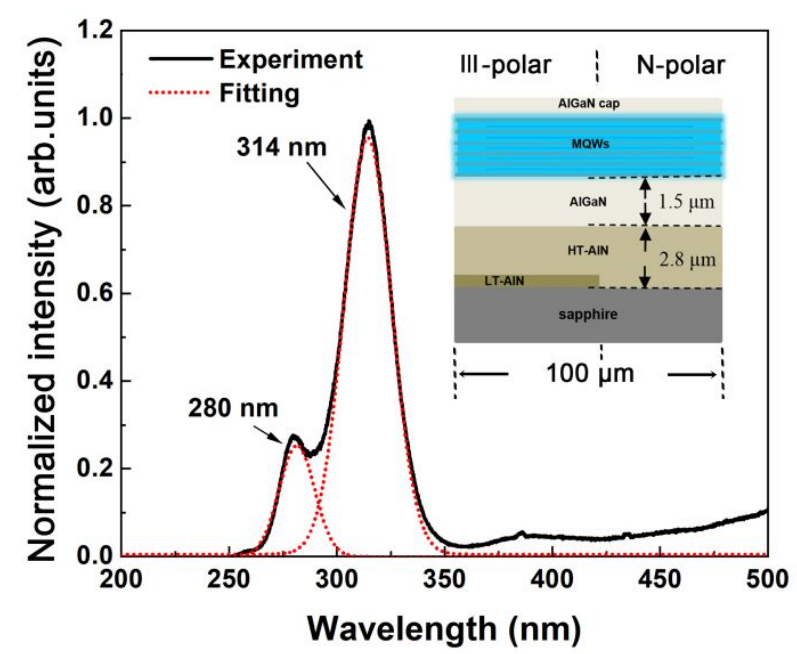

Figure 3. RT-PL spectrum of sample B. Two separate peaks at wavelength of $280 \mathrm{~nm}$ and $314 \mathrm{~nm}$ are deconvoluted. The inset image shows the schematic structure of LPS MQW.

As shown above, large peak separation of more than $30 \mathrm{~nm}$ was observed in LPS-MQWs. In order to clarify the origin of the peak separation, 
$\mu$-cathodoluminescence $(\mu-\mathrm{CL})$ near the inversion domain boundary (IDB) of the AlGaN-based UVC MQWs was performed and shown in Figure 4. The SEM image of the IDB region is shown in Figure 4(a), where N-polar domain is located at the left side, and III-polar domain lies on the right side. The red arrow indicates the direction where CL line-scan was taken. Corresponding CL spectra at different positions across the IDB are stacked vertically in inset image of Figure 4(a). It is obvious that III-polar domain is dominant with single peak emission of $260 \mathrm{~nm}$, and N-polar domain is dominant with a broad emission peak larger than $310 \mathrm{~nm}$. The shorter wavelength of CL emission compared to the corresponding PL emission at $280 \mathrm{~nm}$ can be explained by the band filling effect due to much higher excitation electron energy amid CL experiments ${ }^{12}$. Moreover, CL intensities in N-polar domain are stronger than those in III-polar domains, which is consistent with the prominent longer-wavelength peak revealed in Figure 3. Note that the enhanced luminescence intensity observed in N-polar domain is similar to that of LPS-based UVA MQW, ${ }^{10}$ in which case light scatters in V-shape defect and 3D carrier transport with efficient recombination are the two main reasons. ${ }^{12}$ However, there are two observations that are out of our expectation. Firstly, near the domain boundaries, no luminescence enhancement is identified, which is in contrast to our previous results. ${ }^{12}$ This could possibly originate from worse crystalline quality of MQWs and non-abrupt interface. Secondly, a strong peak separation of more than $30 \mathrm{~nm}$ is observed between III- and N-polar domains of LPS. To further investigate the underlying mechanism of the latter observation, monochromatic CL mapping images were taken at wavelength of 
$314 \mathrm{~nm}$ as shown in Figure 4(b). Many quantum-dots (QDs)-like bright spots are observed in the N-polar domains. A closer look on the monochromatic CL intensity mapping in Figure 4(a) as well as its corresponding SEM image in Figure 4(c) indicats that the bright spots with red squares are distributed randomly on the surface, showing no correlation with the surface morphology. Therefore, in-depth nanoscale structure analysis is highly desired.
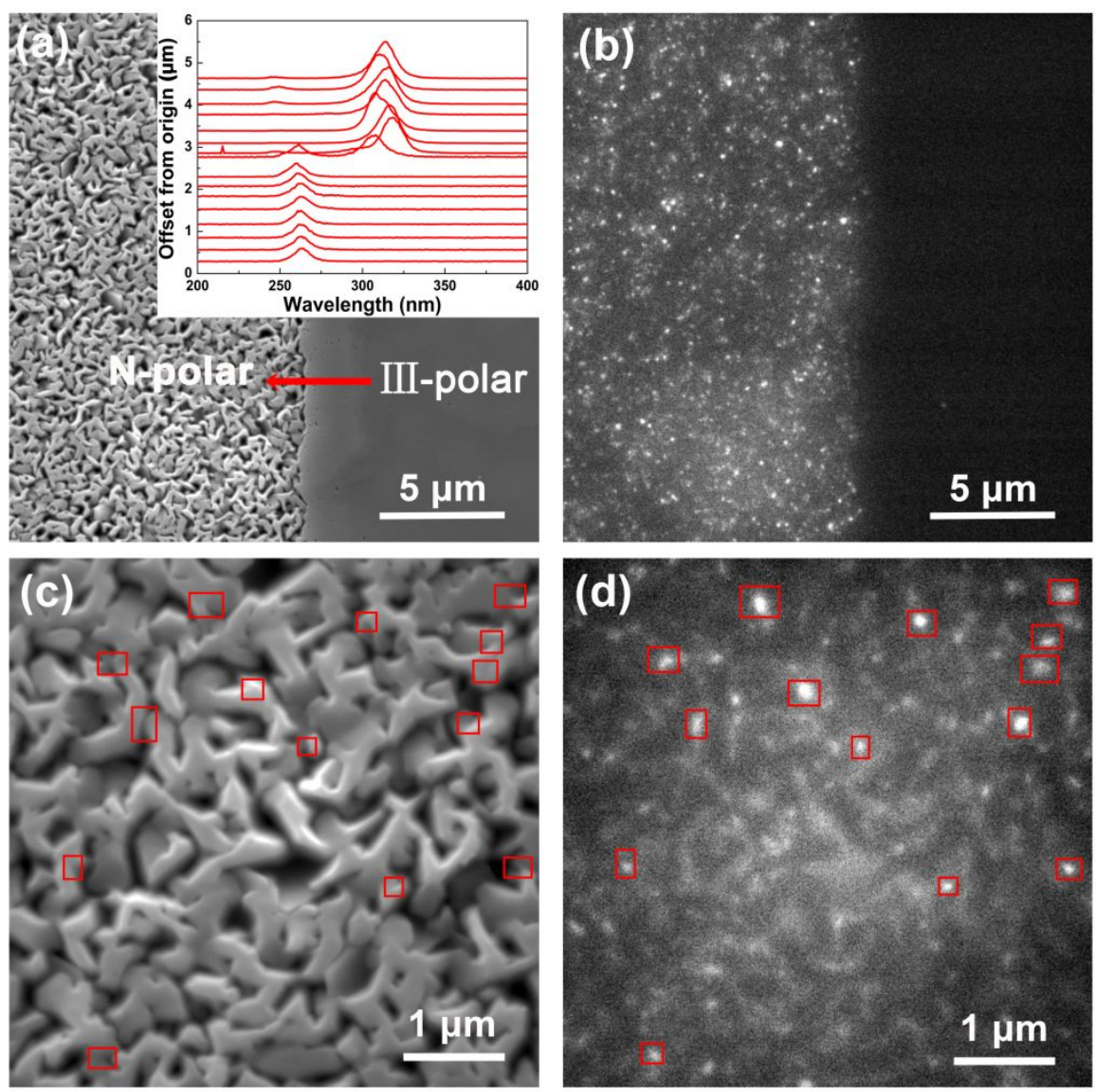

Figure 4. Top-view SEM image of sample B near IDB, where the CL line scan direction is denoted by the red arrow and the CL spectra are stacked in the inset image (a); monochromatic CL intensity map at $314 \mathrm{~nm}$ (b); Zoom-in SEM of the N-polar domain (c) and corresponding CL intensity distribution (d) where the bright luminescence spots are denoted by the red boxes. 
The microstructure of the LPS-based AlGaN MQWs was revealed via a high-angle annular dark-field imaging-scanning transmission electron microscopy (HAADF-STEM). Figure 5(a) shows the detailed structure from high temperature (HT)-AlN layer to the sample surface. III-polar and N-polar domains are located on the left and right side, respectively. The HT-AlN and AlGaN layers can be easily distinguished by the contrast difference. ${ }^{20}$ III-polar domain on the left hand side of Figure 5(a) has homogeneous and sharp interface. Owing to smaller surface diffusion length and consequently 3-D growth mode in N-polar surfaces, ${ }^{21}$ semi-polar MQWs are formed on the slanted sidewalls of the V-shaped or truncated pyramids. III- and $\mathrm{N}$-polar domains are separated by the IDB region of approximately $300 \mathrm{~nm}$ wide, with a V-shape defect located on top. The crystalline quality of N-polar domain is much worse than that of III-polar domain, as N-polar domain is dominated with many V-defects and threading dislocations marked by the orange arrows. This is consistent with the result of HRXRD RC characterization shown above. The zoom-in views of the MQWs in III- and N-polar domains are shown in Figure $\mathbf{5}(\mathbf{b}, \mathbf{d})$ and $(\mathbf{c}, \mathbf{e})$, respectively. Smooth MQWs with clear interfaces are observed in III-polar domain in Figure 5(b) and (d). The quantum well (QW) and quantum barrier (QB) thicknesses are $\sim 2.8$ and $\sim 8 \mathrm{~nm}$, respectively, in accordance with the targeted values. For N-polar domains in Figure 5(c), MQW thickness is non-uniform. A zoom-in view in Figure 5(e) reveals that compared to III-polar domain, the thicknesses of QW is broadened to 3.5-4 $\mathrm{nm}$ in the planar region of N-polar domain. In V-trench regions where semipolar planes are dominant, QW and QB thicknesses increase even larger. The thicker QW 
in N-polar domain is consistent with the PL/CL redshift due to weaker quantum confinement. The increase in thickness of QWs observed on the nanoscale V-pits may be due to atoms lateral diffusion that gradually fills the V-pits, resulting in an increase in QW thickness and consequently red shift in PL/CL.
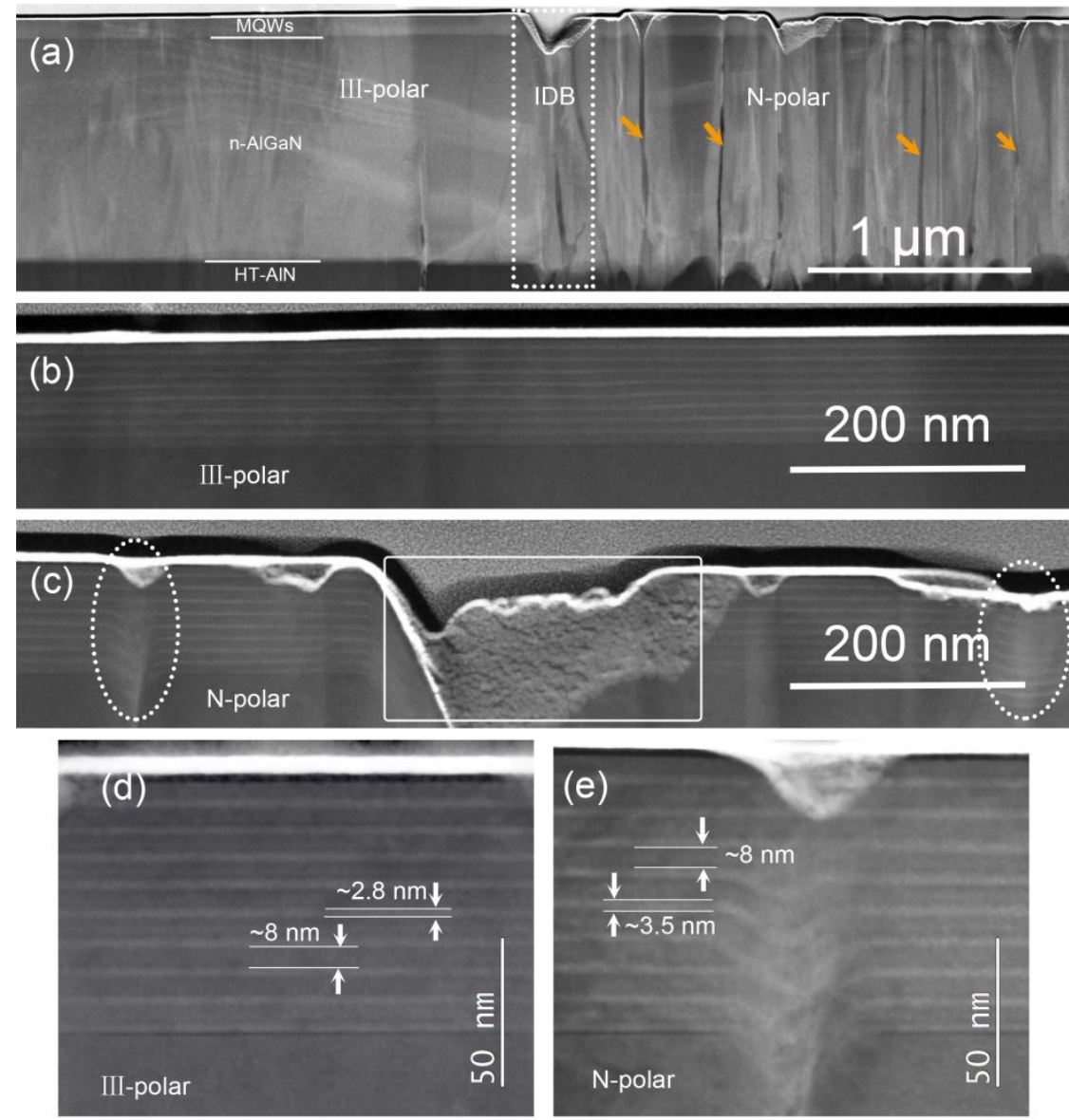

Figure 5. Low magnification cross-sectional HAADF STEM image of sample B (a). Zoom-in views of the LPS-based UVC MQWs regions in III-polar domains (b) (d) and N-polar domains (c) (e).

To simply clarify the contribution of QW broadening to the peak shift, a structure model was developed to simulate the optical behavior of such QW by solving Schrodinger Poisson equations self consistently. ${ }^{22}$ To reduce calculation workload, 
two $\mathrm{Al}_{0.55} \mathrm{Ga}_{0.45} \mathrm{~N} / \mathrm{Al}_{0.4} \mathrm{Ga}_{0.6} \mathrm{~N}$ single quantum well (SQW) LEDs with $\mathrm{QW}$ width of $2.8 \mathrm{~nm}$ and $3.8 \mathrm{~nm}$ were analyzed, representing the case in III- and N-polar domain, respectively. Schematic structure and simulated emission spectra are shown in Figure S2. When the QW width is broadened from $2.8 \mathrm{~nm}$ to $3.8 \mathrm{~nm}$, a negligible $3 \mathrm{~nm}$ red shift is observed, suggesting that thickness broadening does not play a significant role in peak separation observed in experiment. The large peak shift over $30 \mathrm{~nm}$ between III- and N-polar domains is expected to originate from composition inhomogeneity and thus carrier localization effect.

Another phenomenon that worth noting is that there are two kinds of V-trenches as shown in the N-polar domain in Figure 5(c): the first type is marked by the white solid box, where QW is interrupted, and the second type is marked by the dotted circles, where MQW heterojunctions are blurred. Larger incorporation of Ga inside the semi-polar surface is observed according to the contrast difference as revealed by STEM image in Figure 5(e), leading to Ga-enrichment and carrier localization in $\mathrm{N}$-polar domains. In summary, it is expected that the first kind of V-trench will hinder the lateral migration of carriers, whereas the second type promotes radiative recombination with enhanced luminescence intensity. Therefore, the previously observed QD-like luminescence spot in CL mapping is directly correlated with the second type of V-trench.

Optical properties of MQWs are also correlated with strain state. To provide a complete understanding, Raman spectroscopy of LPS is revealed in Figure 6. The modes of $\mathrm{Al}_{2} \mathrm{O}_{3} \mathrm{Eg}$, GaN-like $\mathrm{E}_{2}^{\text {high }}$, AlN-like $\mathrm{E}_{2}^{\text {high }}$, and $\mathrm{AlGaN} \mathrm{A}_{1}{ }^{\mathrm{LO}}$ are clearly 
identified in the spectra. The AlN-like $\mathrm{E}_{2}{ }^{\text {high }}$ mode can be used to estimate the strain of the films if the position of strain-free AlN at $656 \mathrm{~cm}^{-1}$ is considered. ${ }^{23}$ The zoom-in view of the $\mathrm{AlN}$-like $\mathrm{E}_{2}^{\text {high }}$ mode in the inset image shows that the Raman scattering peak is located at $652.5 \mathrm{~cm}^{-1}$ in the III-polar domain, indecating a tensile strain of 1.16 Gpa, and at $658.7 \mathrm{~cm}^{-1}$ in the N-polar domain, suggesting a compressive strain of 0.9 Gpa. ${ }^{24}$ However, it is widely accepted that compressive strain in the UV MQW leads to blue shift of the peak position due to strain-induced composition pulling effect. ${ }^{25}$ This is in contrast with the PL spectra shown in this work, that N-polar domain has longer emission wavelength. Overall a conclusion can be safely drawn that thickness fluctuation and composition inhomogeneity in N-polar domain are more critical in determining the optical property of LPS.

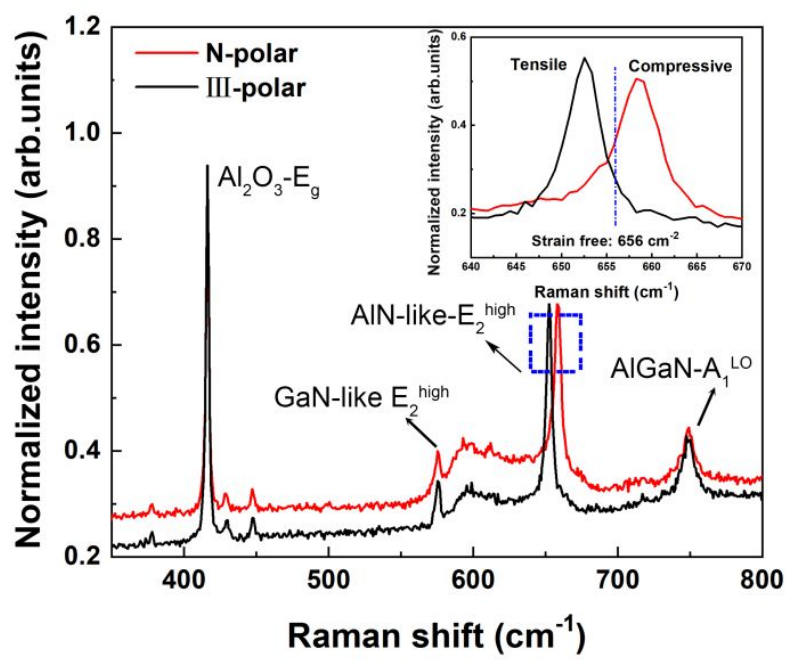

Figure 6. Raman spectra of III and N-polar domains in Sample B. The inset image shows the zoom-in view of the Raman shift of AlN-like- $E_{2}{ }^{\text {high }}$ mode with different polarity, and vertical dashed line is the strain-free position of AlN. 
Even though optimized nitridation condition was performed on sample B, luminescence enhancement in the IDB region was not obtained as previously reported by Fiorentini and Kirste. ${ }^{26,} 27$ It is suspected that the discrepancy can be induced by the rough surface of the MQWs in N-polar domains. Carriers will encounter large transporting barriers across the IDBs due to rugged interface or even get blocked by the V-trenches shown in Figure 5, counteracting the benefits from carrier localization effect near the IDBs. Therefore, sample B proves to have no advantage in enhancing the luminescence with respect to conventional uniform III-polar MQWs. Moreover, the occurrence of peak separation in LPS greatly hinders the wide application of UVC LEDs. Take UV disinfection as an example, the efficiency is strongly dependent on the wavelength, while double peaks have no benefits but only lead to deterioration of the efficiency in killing a certain type of bacteria. Therefore, in order to obtain a single peak emission, surface morphology of N-polar domain needs to be further modified.

It has been reported that, insertion of AlGaN superlattice (SL) beneath the MQWs can act as strain-relief layer as well as dislocation filter, leading to improved crystalline quality and better optical property. ${ }^{28}$ Additionally, surface morphologies of N-polar thin films can also be greatly improved by raising V/III ratio and growth temperature. $^{29,} 30$ In a newly prepared LPS-based UVC MQW sample D, same annealing and nitridation conditions as sample B were utilized, but V/III ratio was increased from 600 to 2000, and fifty pairs of AlGaN/AlN SL were inserted between HT-AlN and $\mathrm{Al}_{0.6} \mathrm{Ga}_{0.4} \mathrm{~N}$ transition layer. Corresponding cross sectional HAADF 
STEM images are shown in Figure S3. Especially, the N-polar domain was sliced from a V-defect area of sample D in order to investigate the contribution of SL. After inserting fifty pairs of SL, many defects were annihilated under AlGaN template in N-polar domain, thus a relatively better crystalline quality and flat surface were obtained. The zoom-in view of the MQWs across the main V-defect area in N-polar domain of sample D is shown in Figure S3 (d). Obviously, there is no obvious Ga-enrichment regions or thickness fluctuations like in sample B, suggesting that improved surface morphology and composition homogeneity are the key parameters to achieve enhanced light output near the IDB. Table 1 summarized the growth conditions, etching susceptibility and purpose during the experimental design.

Table 1 Growth conditions and characteristic properties of LPS-based UVC-MQWs.

\begin{tabular}{cccccc}
\hline \multirow{2}{*}{ samples } & $\begin{array}{c}\text { Nitridation } \\
\text { condition }\end{array}$ & $\begin{array}{c}\mathrm{V} / \mathrm{II} \\
\text { ratio }\end{array}$ & $\begin{array}{c}\text { Wet etch } \\
\text { susceptibility }\end{array}$ & Conclusion & purpose \\
\hline \multirow{2}{*}{$\mathrm{A}$} & $950^{\circ} \mathrm{C}$ & \multirow{2}{*}{600} & Hard & $\begin{array}{c}\text { Insufficient } \\
\text { nitridation }\end{array}$ & \\
& $5 \mathrm{~min}$ & & & $\begin{array}{c}\text { Proper } \\
\text { nitridation }\end{array}$ & $\begin{array}{c}\text { Nitridation optimization for } \\
\text { polarity control }\end{array}$ \\
& $1050^{\circ} \mathrm{C}$ & 600 & Easy & Over nitridation & \\
& $5 \mathrm{~min}$ & & Easy & Proper & Eliminate peak separation \\
C & $1150^{\circ} \mathrm{C}$ & 600 & Easy & nitridation & \\
& $5 \mathrm{~min}$ & & & &
\end{tabular}

The top-view SEM image of N-polar domain is illustrated in Figure 7(a), showing a relatively coherent and flat surface morphology. The SEM image of sample B with the same magnification is also shown in Figure 7(b) for comparison purpose. Sample B shows fragmented surface features with large proportion of semi-polar planes, and the surface roughness is $27.3 \mathrm{~nm}$. As a result, MQWs are separated apart into small 
domains, and consequently carries cannot easily laterally transport and efficiently recombine. In contrast, flat and continuous $c$-plane surface with small pits are shown in sample D, and the surface roughness is reduced to $17 \mathrm{~nm}$. The corresponding Atomic Force Microscope (AFM) images of these two samples are illustrated in Figure S4. Figure 7 (c) and (d) illustrate the pseudomorphic 3-D CL intensity distribution for Sample D and B, respectively. $X$-axis represents the lateral distance adjacent to the IDB, and $Y$-axis represents emission wavelength. Color scale-bar on the right shows the normalized CL intensity. In sample D, surprisingly, there is no obvious peak separation between III- and N-polar domains after growth modification, i.e. single peak emission at $275 \mathrm{~nm}$ is achieved. The strongest intensity comes from the IDB region, which is consistent with previous work. ${ }^{27}$ The peak width broadens near the IDB, mainly because of interface roughness and carrier localization effect. A CL line scan at wavelength of $275 \mathrm{~nm}$ across IDB region of sample D is shown in Figure S5. Almost 10-fold emission enhancement is obtained near the IDB with respect to adjacent III- and N-polar domains. Thanks to the relatively coherent and flat N-polar surface of sample D, carries can laterally migrate and effectively radiative recombine encounter along the IDB, as the IDB acts as a radiative recombination center. For sample B, as expected, III- and N-polar domains exhibit two peaks with distinguishable peak separation. Peak width is also broadened in N-polar domain compared to that in III-polar domain due to QW thickness fluctuations and composition inhomogeneity. The discrepancy in the CL spectra between sample B and D further confirms that a relatively flat and coherent surface of N-polar is 
extremely vital to obtain single-peak emission and serve as an important prerequisite to enhance the output power of LPS-based UVC LEDs.
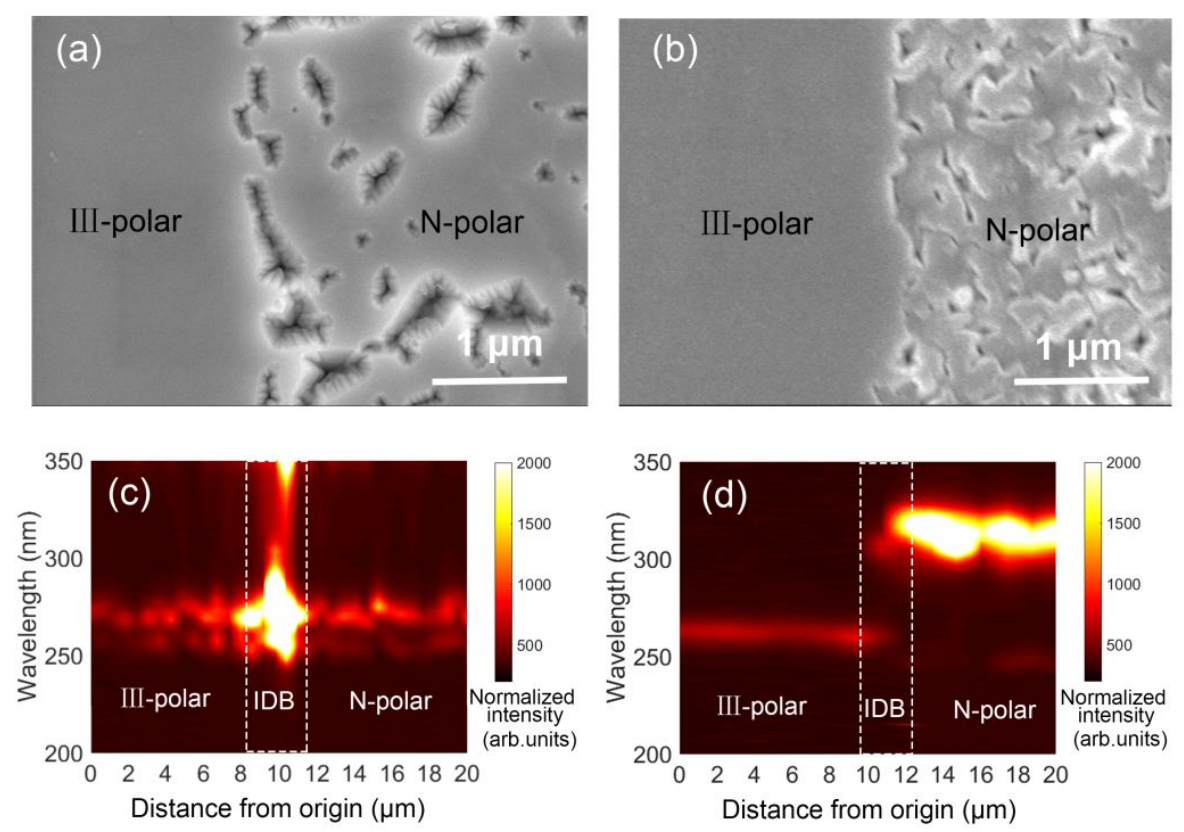

Figure 7. Top-view SEM images of sample D (a) and B (b) with and without inserted SL layer for N-polar surface morphology modification, respectively; Pseudomorphic 3-D CL intensity mapping as a function of lateral distance and wavelength for sample D (C) and B (d), respectively.

\section{Conclusions}

In summary, a novel AlGaN UVC-MQW structure comprising laterally distributed III- and N-polar domains on a single substrate was designed and fabricated. The influences of pre-growth nitridation, V/III ratio and insertion of SL layer on polarity control, surface roughness, and nanoscale optical properties were carefully investigated. $\mu$-CL and cross-sectional HAADF STEM reveal that QW thickness fluctuation and Ga-enrichment are induced inside N-polar domains, leading to 
nanoscale potential minimum and carrier localization and even peak separation between III- and N-polar domains. Further growth optimization leads to a single peak emission at wavelength of $275 \mathrm{~nm}$ for both III- and N-polar domains, and 10-fold enhanced luminescence is observed at the domain boundary. This work lays a solid foundation for the development of novel high-efficiency UVC emitters in the future.

\section{Supporting Information}

Figure S1. FWHM values of HRXRD RC of AlGaN template in N and III-polar domains of Sample B. Figure S2. Schematic structure of SQW LED with QW width of $2.8 \mathrm{~nm}$ and $3.8 \mathrm{~nm}$, respectively (a); Corresponding EL emission spectrum illustrating only $3 \mathrm{~nm}$ peak difference (b). Figure S3. Low magnification cross-sectional HAADF STEM image of sample D in III -polar domain (a) and N-polar domain (b); Zoom-in views of the MQWs of III -polar domain (c) and N-polar domain (d). Figure S4. Topographic AFM images of sample D (a) and sample B (b) in a $20 \times 20 \mu \mathrm{m}^{2}$ region. Figure S5. RT-PL spectrum of sample D (a); CL intensity distribution at wavelength of $275 \mathrm{~nm}$ across IDB region of sample D (b).

\section{Conflicts of interest}

There are no conflicts to declare.

\section{Acknowledgements}

This work was supported by National Key Research and Development Program of China (2016YFB0400802), National Natural Science Foundation of China (61974149, 61704176), Key Research and Development Program of Zhejiang 
Province (2019C01080, 2020C01145), and Ningbo Innovation 2025 Major Project (2018B10088, 2019B10121).

\section{References}

1. Chevremont, A. C.; Boudenne, J. L.; Coulomb, B.; Farnet, A. M., Impact of watering with UV-LED-treated wastewater on microbial and physico-chemical parameters of soil. Water research 2013, 47, 1971-82.

2. Song, K.; Mohseni, M.; Taghipour, F., Application of ultraviolet light-emitting diodes (UV-LEDs) for water disinfection: A review. Water research 2016, 94, 341-349.

3. Takeuchi, T.; Sota, S.; Katsuragawa, M.; Komori, M.; Takeuchi, H.; Amano, H.; Akasaki, I., Quantum-Confined Stark Effect due to Piezoelectric Fields in GaInN Strained Quantum Wells. Japanese Journal of Applied Physics 1997, 36, L382-L385.

4. David, A.; Fujii, T.; Sharma, R.; McGroddy, K.; Nakamura, S.; DenBaars, S. P.; Hu, E. L.; Weisbuch, C.; Benisty, H., Photonic-crystal GaN light-emitting diodes with tailored guided modes distribution. Applied Physics Letters 2006, 88, 061124.

5. Kneissl, M.; Seong, T.-Y.; Han, J.; Amano, H., The emergence and prospects of deep-ultraviolet light-emitting diode technologies. Nature Photonics 2019, 13, 233-244. 
6. Narukawa, Y.; Ichikawa, M.; Sanga, D.; Sano, M.; Mukai, T., White light emitting diodes with super-high luminous efficacy. Journal of Physics D: Applied Physics 2010, 43, 354002.

7. Dong, P.; Yan, J.; Wang, J.; Zhang, Y.; Geng, C.; Wei, T.; Cong, P.; Zhang, Y.; Zeng, J.; Tian, Y.; Sun, L.; Yan, Q.; Li, J.; Fan, S.; Qin, Z., 282-nm AlGaN-based deep ultraviolet light-emitting diodes with improved performance on nano-patterned sapphire substrates. Applied Physics Letters 2013, 102, 241113.

8. Xu, H.; Jiang, J. a.; Sheikhi, M.; Chen, Z.; Hoo, J.; Guo, S.; Guo, W.; Sun, H.; Ye, J., Single peak deep ultraviolet emission and high internal quantum efficiency in AlGaN quantum wells grown on large miscut sapphire substrates. Superlattices and Microstructures 2019, 129, 20-27.

9. Jiang, J.; Guo, W.; Xu, H.; Yang, Z.; Guo, S.; Xie, W.; Chee, K. W. A.; Zeng, Y.; Ye, J., Performance enhancement of ultraviolet light emitting diode incorporating Al nanohole arrays. Nanotechnology 2018, 29, 45LT01.

10. Guo, W.; Sun, H.; Torre, B.; Li, J.; Sheikhi, M.; Jiang, J.; Li, H.; Guo, S.; Li, K.-H.; Lin, R.; Giugni, A.; Di Fabrizio, E.; Li, X.; Ye, J., Lateral-Polarity Structure of AlGaN Quantum Wells: A Promising Approach to Enhancing the Ultraviolet Luminescence. Advanced Functional Materials 2018, 28, 1802395.

11. Park, M.; Cuomo, J. J.; Rodriguez, B. J.; Yang, W. C.; Nemanich, R. J.; Ambacher, O., Micro-Raman study of electronic properties of inversion domains in 
GaN-based lateral polarity heterostructures. Journal of Applied Physics 2003, 93, 9542-9547.

12. Guo, W.; Mitra, S.; Jiang, J. a.; Xu, H.; Sheikhi, M.; Sun, H.; Tian, K.; Zhang, Z.-h.; Jiang, H.; Roqan, I. S.; Li, X.; Ye, J., Three-dimensional band diagram in lateral polarity junction III-nitride heterostructures. Optica 2019, 6, 1058.

13. Hoffmann, M. P.; Kirste, R.; Mita, S.; Guo, W.; Tweedie, J.; Bobea, M.; Bryan, I.; Bryan, Z.; Gerhold, M.; Collazo, R.; Sitar, Z., Growth and characterization of AlxGa1-xN lateral polarity structures. physica status solidi (a) 2015, 212, 1039-1042.

14. Hite, J., Progress in periodically oriented III-nitride materials. Journal of Crystal Growth 2016, 456, 133-136.

15. Guo, W.; Kirste, R.; Bryan, I.; Bryan, Z.; Hussey, L.; Reddy, P.; Tweedie, J.; Collazo, R.; Sitar, Z., KOH based selective wet chemical etching of AlN, AlxGa1-xN, and GaN crystals: A way towards substrate removal in deep ultraviolet-light emitting diode. Applied Physics Letters 2015, 106, 082110.

16. Sheng-Rui, X.; Yue, H.; Jin-Cheng, Z.; Xiao-Wei, Z.; Yan-Rong, C.; Xin-Xiu, O.; Wei, M.; Da-Chao, D.; Hao, W., The etching of a-plane GaN epilayers grown by metal-organic chemical vapour deposition. Chinese Physics B 2010, 19, 107204. 
17. Sun, Q.; Suk Cho, Y.; Kong, B. H.; Koun Cho, H.; Shine Ko, T.; Yerino, C. D.; Lee, I.-H.; Han, J., N-face GaN growth on c-plane sapphire by metalorganic chemical vapor deposition. Journal of Crystal Growth 2009, 311, 2948-2952.

18. Mohn, S.; Stolyarchuk, N.; Markurt, T.; Kirste, R.; Hoffmann, M. P.; Collazo, R.; Courville, A.; Di Felice, R.; Sitar, Z.; Vennéguès, P., Polarity Control in Group-III Nitrides beyond Pragmatism. Physical Review Applied 5, 054004.

19. Heying, B.; Wu, X. H.; Keller, S.; Li, Y.; Kapolnek, D.; Keller, B. P.; DenBaars, S. P.; Speck, J. S., Role of threading dislocation structure on the $\mathrm{x}$ - ray diffraction peak widths in epitaxial GaN films. Applied Physics Letters 1996, 68, 643-645.

20. Sun, H.; Mitra, S.; Subedi, R. C.; Zhang, Y.; Guo, W.; Ye, J.; Shakfa, M. K.; Ng, T. K.; Ooi, B. S.; Roqan, I. S.; Zhang, Z.; Dai, J.; Chen, C.; Long, S., Unambiguously Enhanced Ultraviolet Luminescence of AlGaN Wavy Quantum Well Structures Grown on Large Misoriented Sapphire Substrate. Advanced Functional Materials 2019, 1905445.

21. Wang, X.; Zhang, X.; Zhang, H.; Zhao, J.; Wu, Z.; Dai, Q.; Wang, S.; Hu, G.; Cui, Y., Influence of nitridation process on characteristics of N-polar AlGaN films grown by MOCVD. Materials Science in Semiconductor Processing 2017, 64, $147-151$.

22. Chee, K. W. A.; Guo, W.; Wang, J. R.; Wang, Y.; Chen, Y.-e.; Ye, J., Tuning photonic crystal fabrication by nanosphere lithography and surface treatment of 
AlGaN-based ultraviolet light-emitting diodes. Materials \& Design 2018, 160, 661-670.

23. Kirste, R.; Mita, S.; Hussey, L.; Hoffmann, M. P.; Guo, W.; Bryan, I.; Bryan, Z.; Tweedie, J.; Xie, J.; Gerhold, M.; Collazo, R.; Sitar, Z., Polarity control and growth of lateral polarity structures in AlN. Applied Physics Letters 2013, 102, 181913.

24. Goñi, A. R.; Siegle, H.; Syassen, K.; Thomsen, C.; Wagner, J. M., Effect of pressure on optical phonon modes and transverse effective charges inGaNandAlN. Physical Review B 2001, 64.

25. Xu, H.; Long, H.; Jiang, J.; Sheikhi, M.; Li, L.; Guo, W.; Dai, J.; Chen, C.; Ye, J., Strain modulated nanostructure patterned AlGaN-based deep ultraviolet multiple-quantum-wells for polarization control and light extraction efficiency enhancement. Nanotechnology 2019, 30, 435202.

26. Fiorentini, V., Origin of the efficient light emission from inversion domain boundaries in GaN. Applied Physics Letters 2003, 82, 1182-1184.

27. Kirste, R.; Collazo, R.; Callsen, G.; Wagner, M. R.; Kure, T.; Sebastian Reparaz, J.; Mita, S.; Xie, J.; Rice, A.; Tweedie, J.; Sitar, Z.; Hoffmann, A., Temperature dependent photoluminescence of lateral polarity junctions of metal organic chemical vapor deposition grown GaN. Journal of Applied Physics 2011, 110, 093503. 
28. Chen, C. Q.; Zhang, J. P.; Gaevski, M. E.; Wang, H. M.; Sun, W. H.; Fareed, R. S. Q.; Yang, J. W.; Khan, M. A., AlGaN layers grown on GaN using strain-relief interlayers. Applied Physics Letters 2002, 81, 4961-4963.

29. Hite, J. K.; Zoll, R.; Mastro, M. A.; Eddy, C. R., Role of growth parameters in equalizing simultaneous growth of $\mathrm{N}$ - and Ga-polar GaN by MOCVD. physica status solidi (c) 2014, 11, 458-461.

30. Jiang, J.; Zhang, Y.; Yang, F.; Huang, Z.; Yan, L.; Li, P.; Chi, C.; Zhao, D.; Zhang, B.; Du, G., Study of N-polar GaN growth with a high resistivity by metal-organic chemical vapor deposition. Vacuum 2015, 119, 63-67.
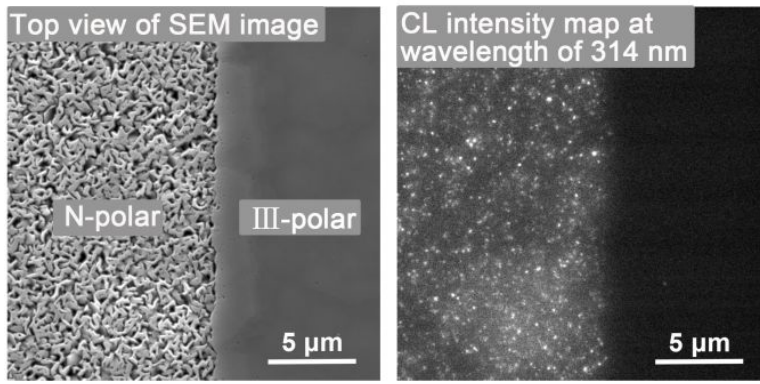

TOC 


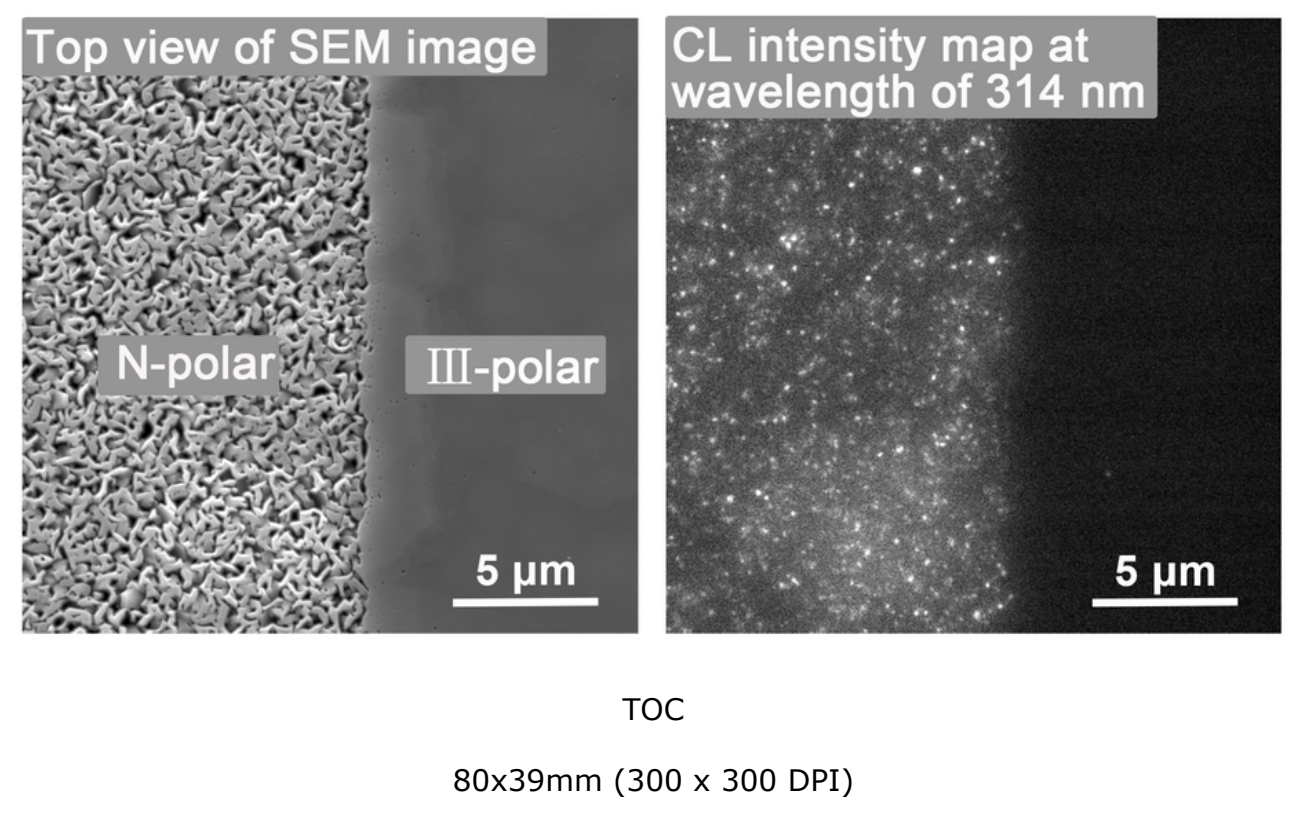

ACS Paragon Plus Environment 


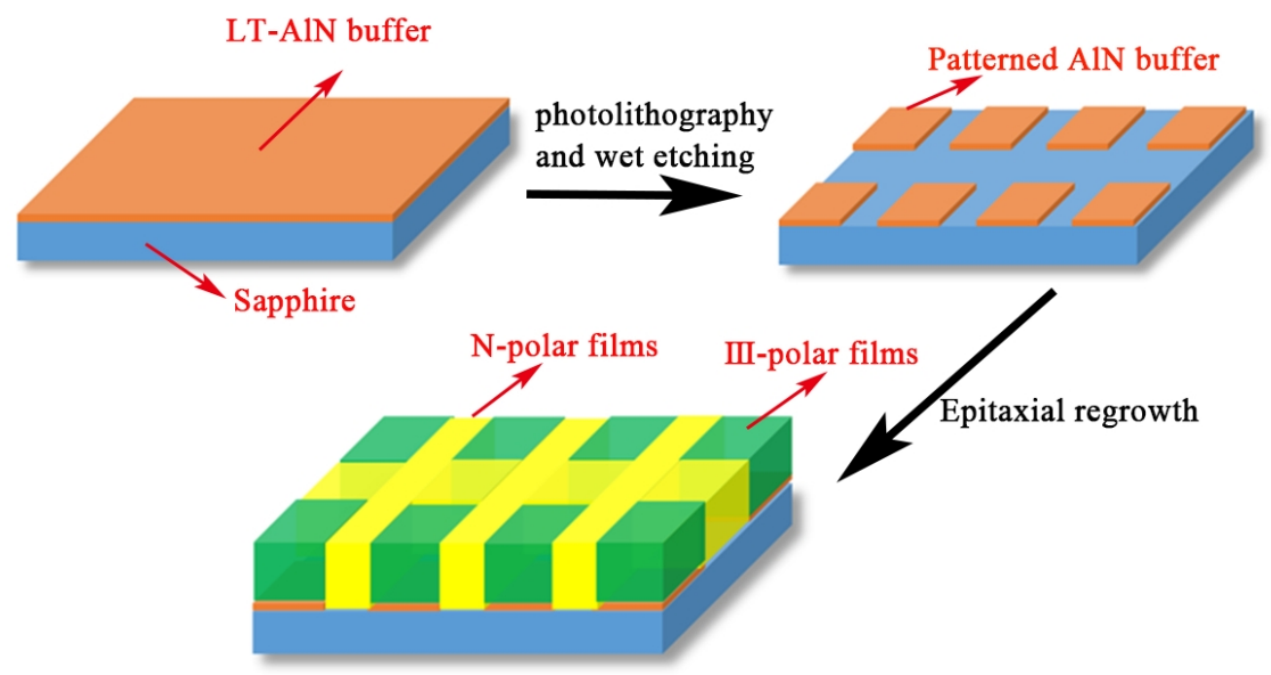

Figure 1. The fabrication process scheme of LPS-based MQWs. The process contains three main steps. Step 1: MOCVD deposition of LT-AIN buffer on c-plane sapphire. Step 2: Fabricating patterned buffer through photolithography and wet etching. Step 3: The patterned substrate is reloaded into the MOCVD chamber for epitaxial regrowth. 

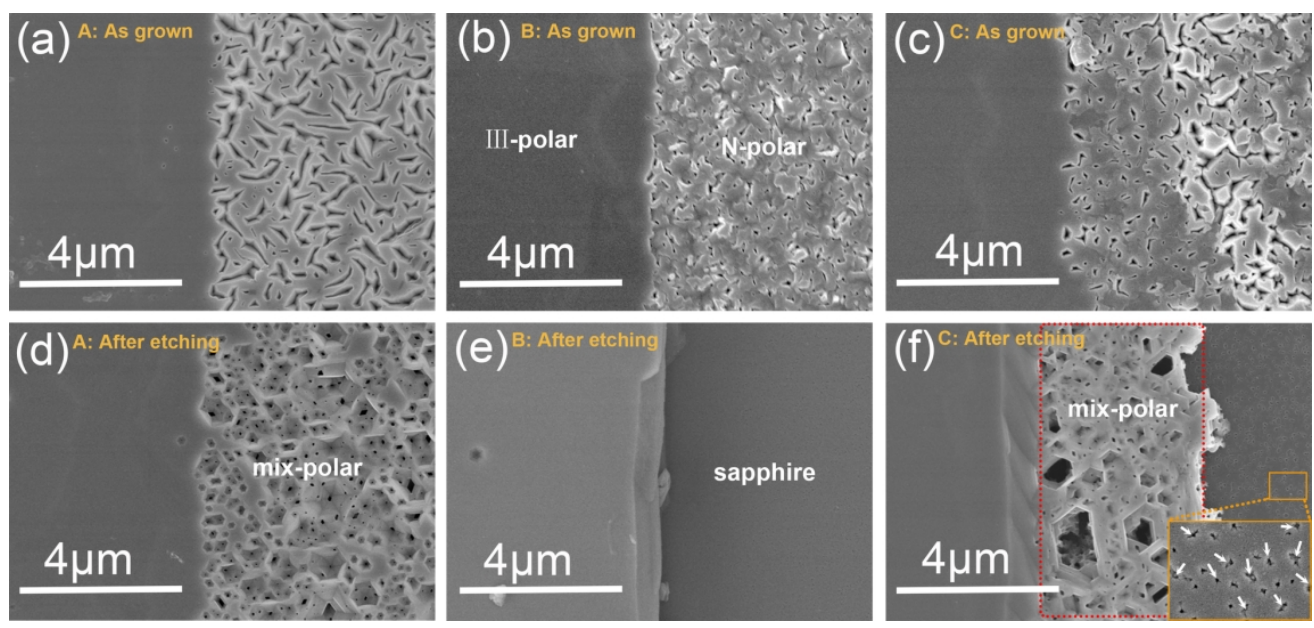

Figure 2. Top-view SEM images of sample A, B and C before (a-c) and after (d-f) KOH etching for 10 min. The left side of each image is III-polar domain, and right side of each image is designated for N-polar domain.

$165 \times 77 \mathrm{~mm}(300 \times 300 \mathrm{DPI})$ 


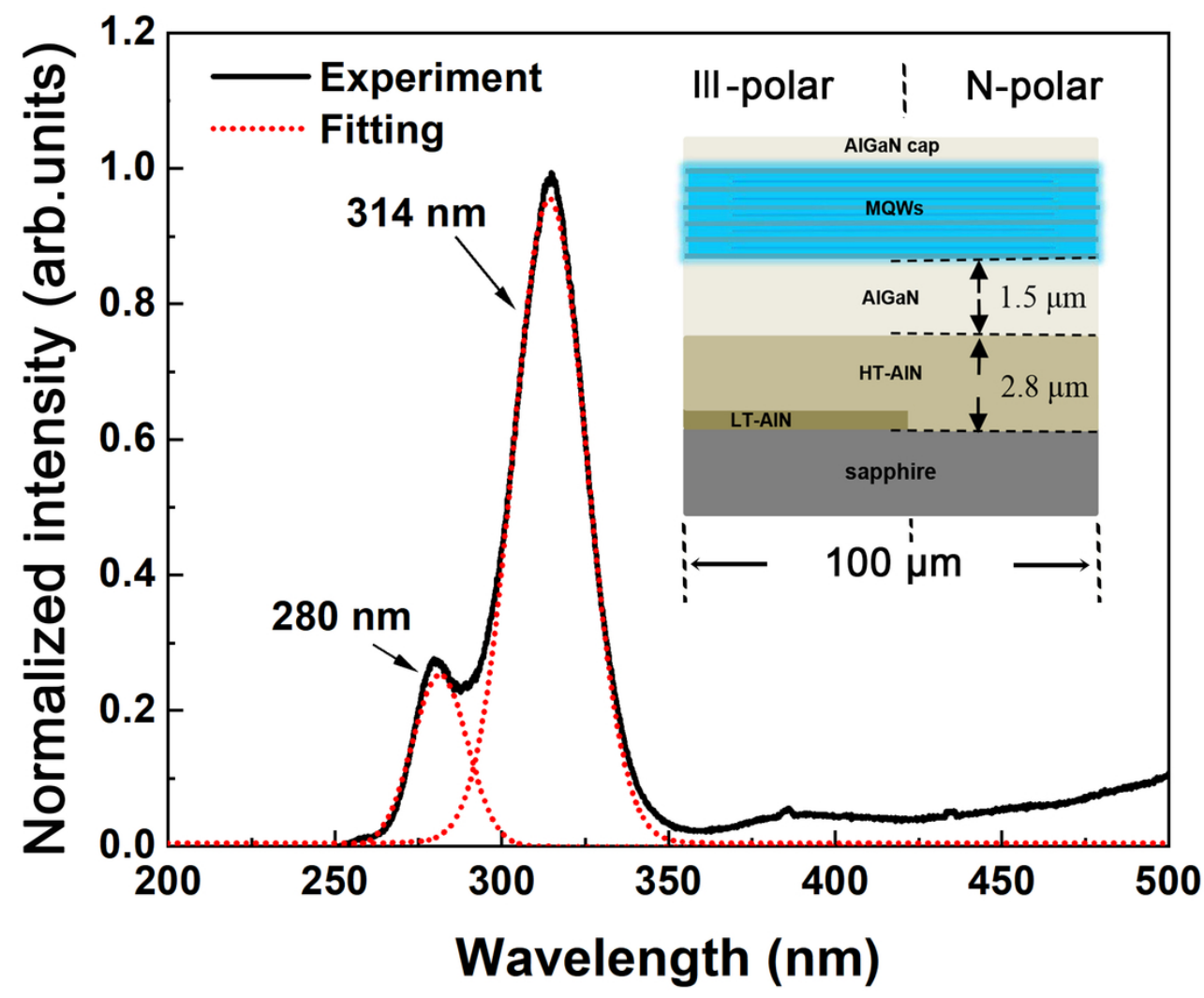

Figure 3. RT-PL spectrum of sample B. Two separate peaks at wavelength of $280 \mathrm{~nm}$ and $314 \mathrm{~nm}$ are deconvoluted. The inset image shows the schematic structure of LPS MQW.

$95 \times 77 \mathrm{~mm}(300 \times 300 \mathrm{DPI})$ 

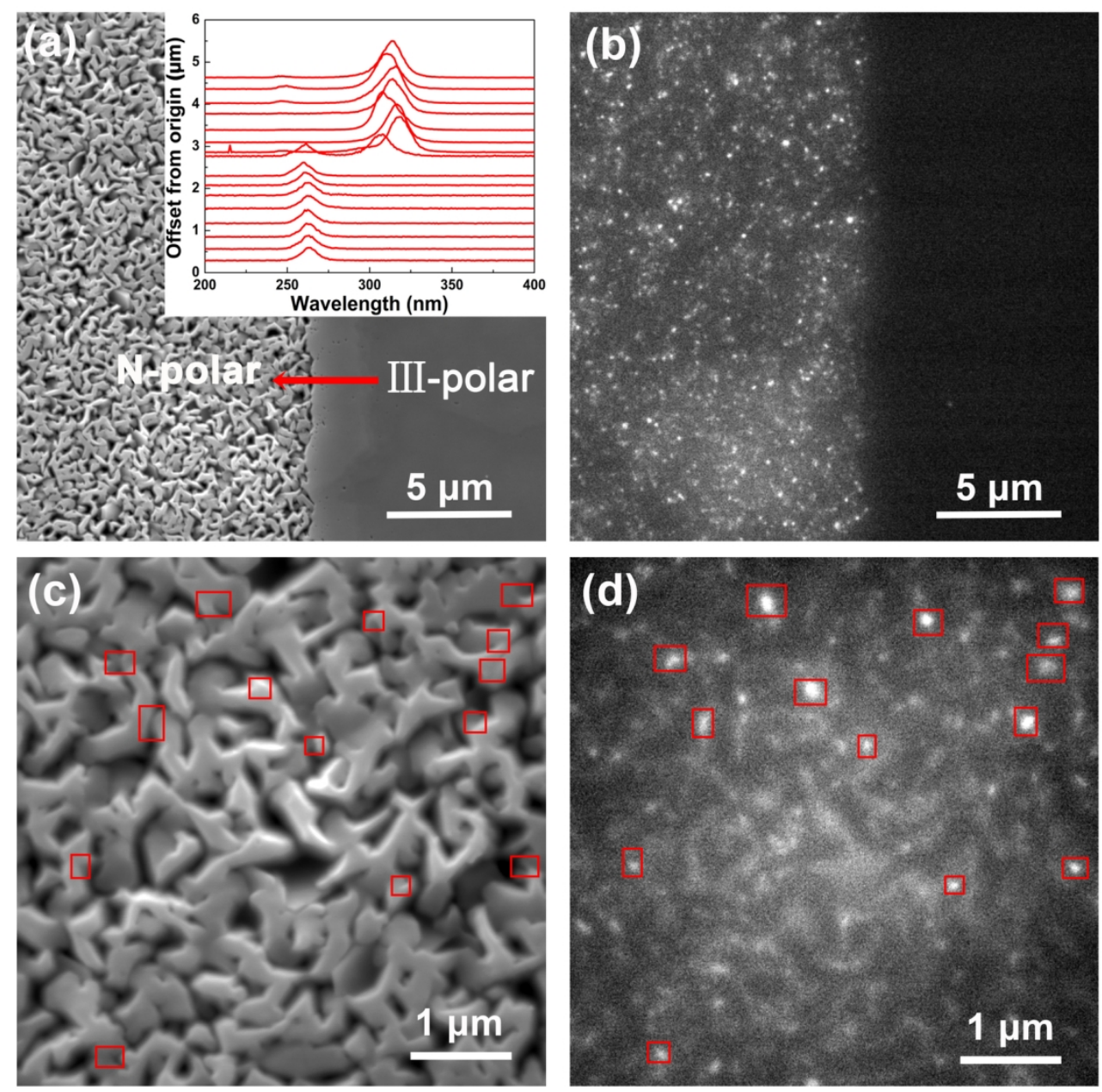

Figure 4. Top-view SEM image of sample B near IDB, where the CL line scan direction is denoted by the red arrow and the CL spectra are stacked in the inset image (a); monochromatic CL intensity map at $314 \mathrm{~nm}$ (b); Zoom-in SEM of the N-polar domain (c) and corresponding CL intensity distribution (d) where the bright luminescence spots are denoted by the red boxes.

$139 \times 138 \mathrm{~mm}(300 \times 300 \mathrm{DPI})$ 


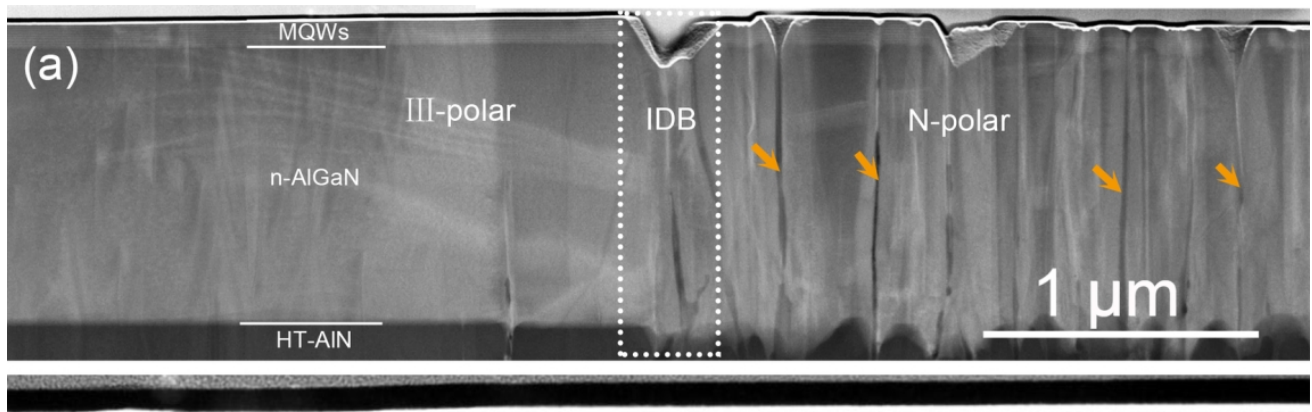

(b)

\section{$200 \mathrm{~nm}$}

III-polar
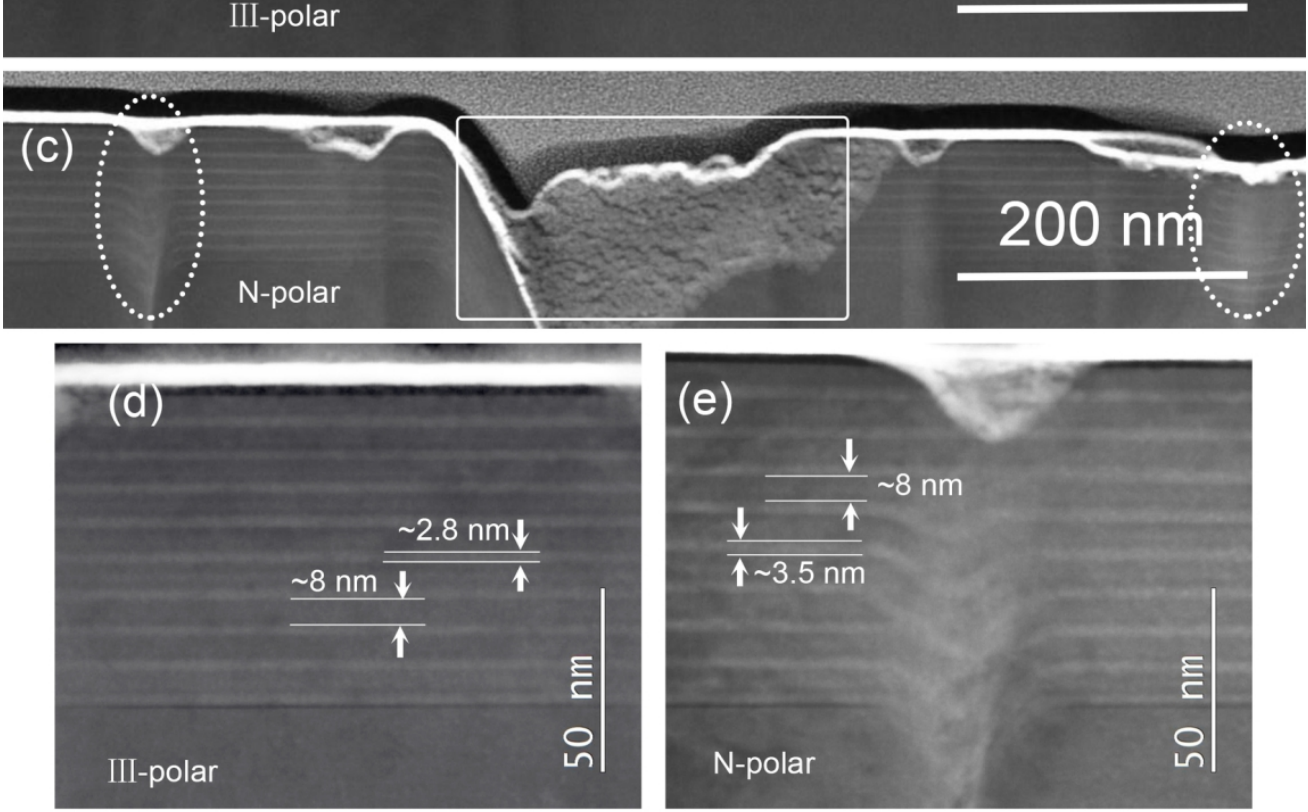

Figure 5. Low magnification cross-sectional HAADF STEM image of sample $B$ (a). Zoom-in views of the LPSbased UVC MQWs regions in III-polar domains (b) (d) and N-polar domains (c) (e).

$139 \times 148 \mathrm{~mm}(300 \times 300$ DPI $)$ 


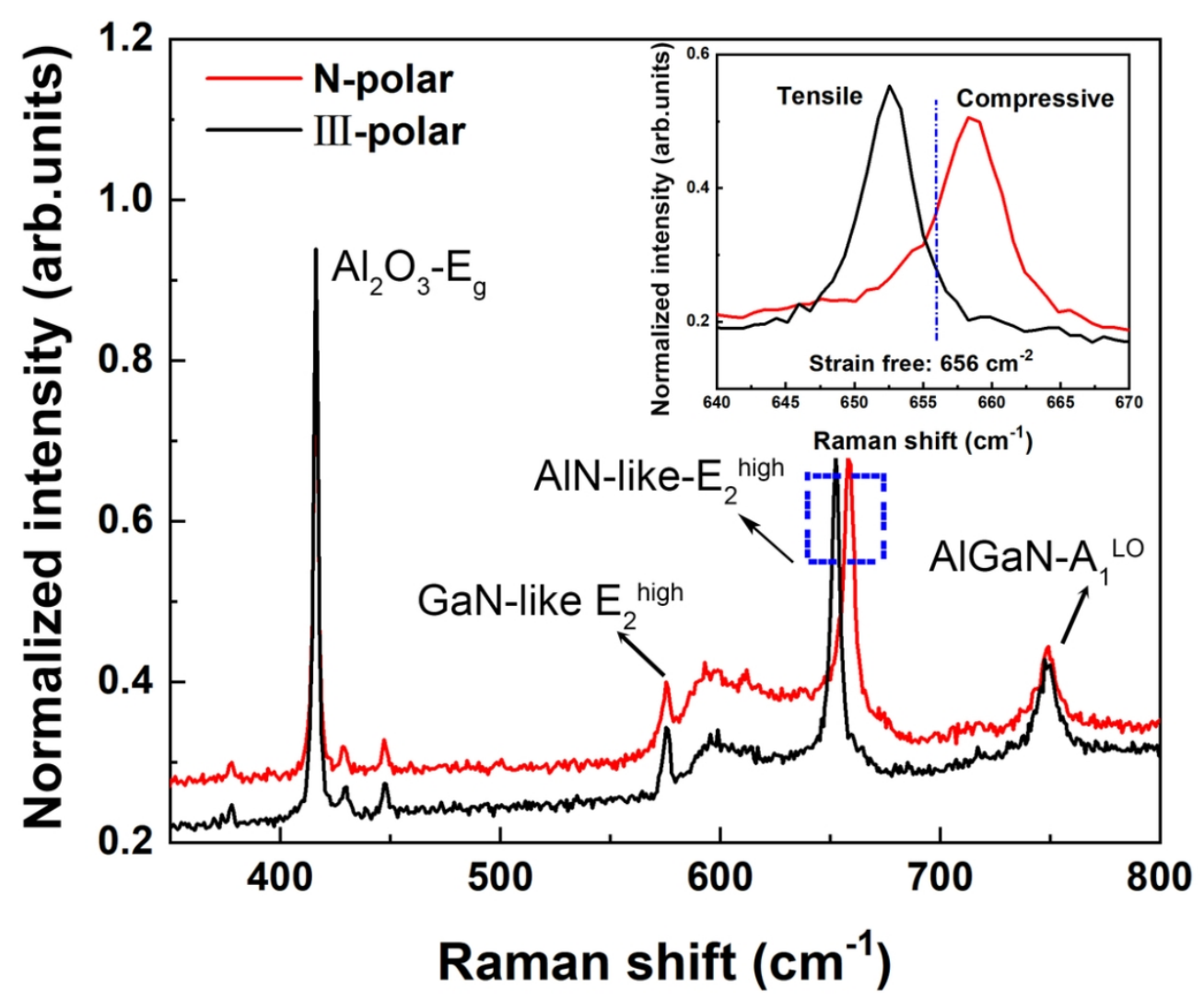

Figure 6. Raman spectra of III and N-polar domains in Sample B. The inset image shows the zoom-in view of the Raman shift of AIN-like-E2high mode with different polarity, and vertical dashed line is the strain-free position of AIN.

$99 \times 83 \mathrm{~mm}(300 \times 300 \mathrm{DPI})$ 

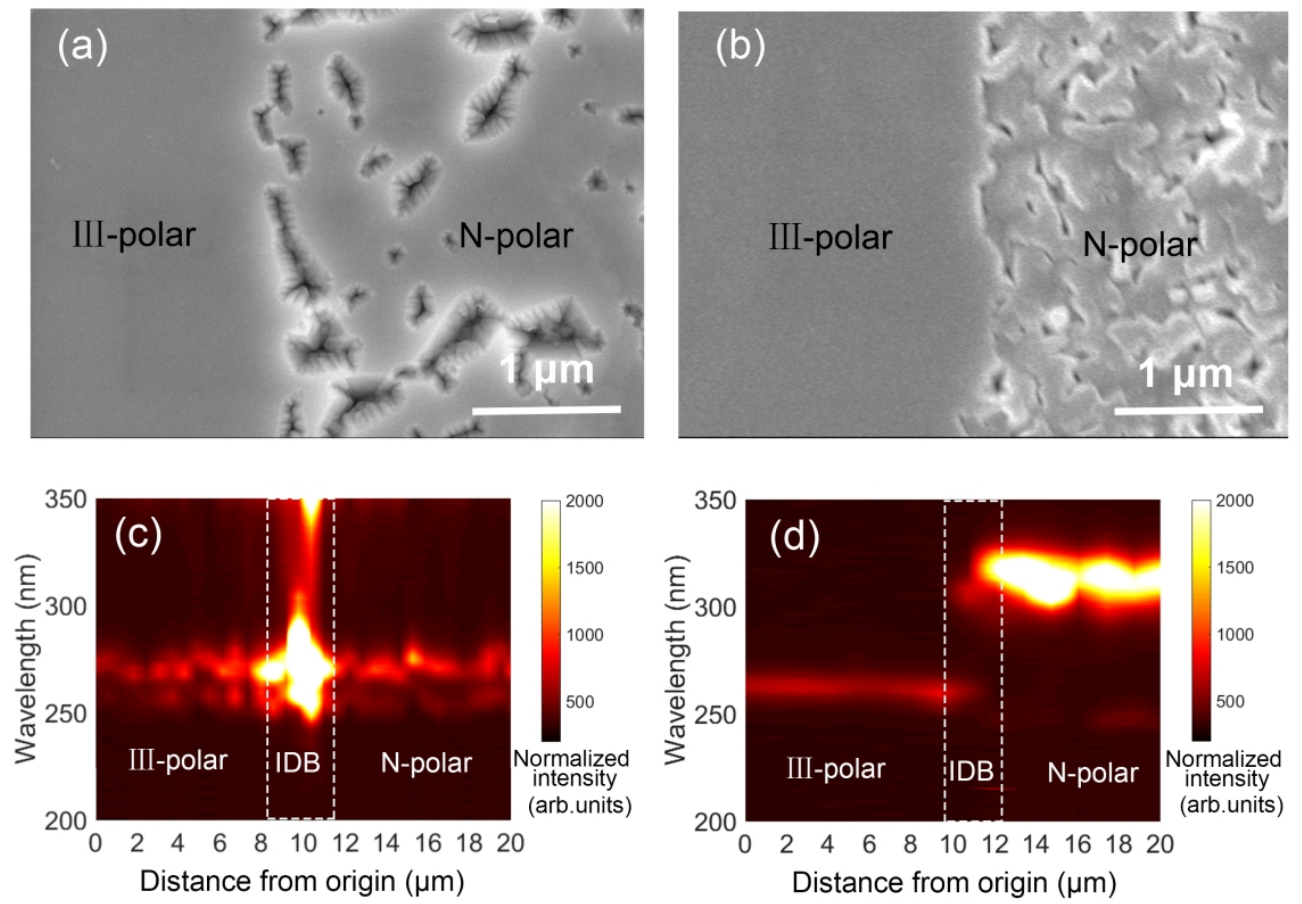

Figure 7. Top-view SEM images of sample D (a) and B (b) with and without inserted SL layer for N-polar surface morphology modification, respectively; Pseudomorphic 3-D CL intensity mapping as a function of lateral distance and wavelength for sample D (C) and B (d), respectively. 